Emina Petrović $\bowtie$

Danijela Ristić Durrant

Miloš Simonović

Žarko Ćojbašić

Vlastimir Nikolić

https://doi.org/10.21278/TOF.453024420

ISSN 1333-1124

eISSN 1849-1391

\title{
VISION-BASED INSPECTION OF TYRE TREAD DEPTH
}

\begin{abstract}
Summary
In this paper, an approach for visual, non-contact automatic inspection of tyre tread depth based on existing image processing techniques is presented. Histograms of oriented gradient are used for feature extraction from images. In order to analyse which set of features gives the best classification results, a linear support-vector machine classifier was trained and tested using different numbers of pixels and numbers of cells per block. The obtained processing and experimental results are presented in this paper.
\end{abstract}

Key words: $\quad$ Histogram of oriented gradient, Support vector machine, Tyre tread depth inspection, Optimal descriptor configuration, Classification

\section{Introduction}

Tyres present one of the most important components in automotive industry. The tyres directly influence the performance and handling of the vehicle, especially the braking system because they represent the only parts of the car that have a direct contact with the road. In many papers, authors studied the influence of tyre characteristics, such as wheel diameter, tyre tread depth, and pressure, on the vehicle behaviour $[1,2,3,4,5,6]$.

Tyres represent the most active safety equipment of any car. Improperly maintained and defective tyres have negative effects on the vehicle acceleration, braking, steering, and comfort, thus causing thousands of deaths. Because of aging and exposure to harsh environments, tyres lose the ability to grip, which leads to an increased a risk of accidents [7].

The condition of tyres is usually inspected during maintenance operations, but vehicle owners should also perform the inspection of tyre tread, tyre pressure as well as the tyre defects. Although the tyre condition is crucial for safety and generally for the vehicle performance, the vast majority of drivers do not monitor the state of tyres and are unaware of the risk if they continue to use their vehicles without ongoing inspections of tyres and ignore the need for the tyre replacement [8].

Traditionally, the tyre inspection is done manually, visually or with some inspection tools; thus, the development of an "easy-to-use" system for automatic visual tyre inspection would 
improve the detection of improper tyres and probably raise awareness about the need for regular tyre replacement.

The tyre tread depth is a vertical distance from the top of the tyre tread to the bottom of the deepest grooves. The main role of the tyre tread is to increase friction between the tyre and the road surface. In addition, the tread also removes water between the tyre and the road surface, providing high friction even in wet conditions and reducing the risk of aquaplaning [9]. As the tyre treads increase safety, a legal minimum of tyre depth is required. If the tyre tread depth is low, there is a higher risk of an accident.

The legal tyre tread depth for cars in Europe is $1.6 \mathrm{~mm}$, but many experts believe that, for safety reasons, the depth of $1.6 \mathrm{~mm}$ is insufficient. Tests performed by independent experts at the Motor Industry Research Association in the United Kingdom (Fig.1) demonstrate that reduced tread depths dramatically increase the wet braking distance of the vehicle. Even though $1.6 \mathrm{~mm}$ of tread depth is the legal minimum in Europe, tread depths of less than $3 \mathrm{~mm}$ considerably increase the safety risk.

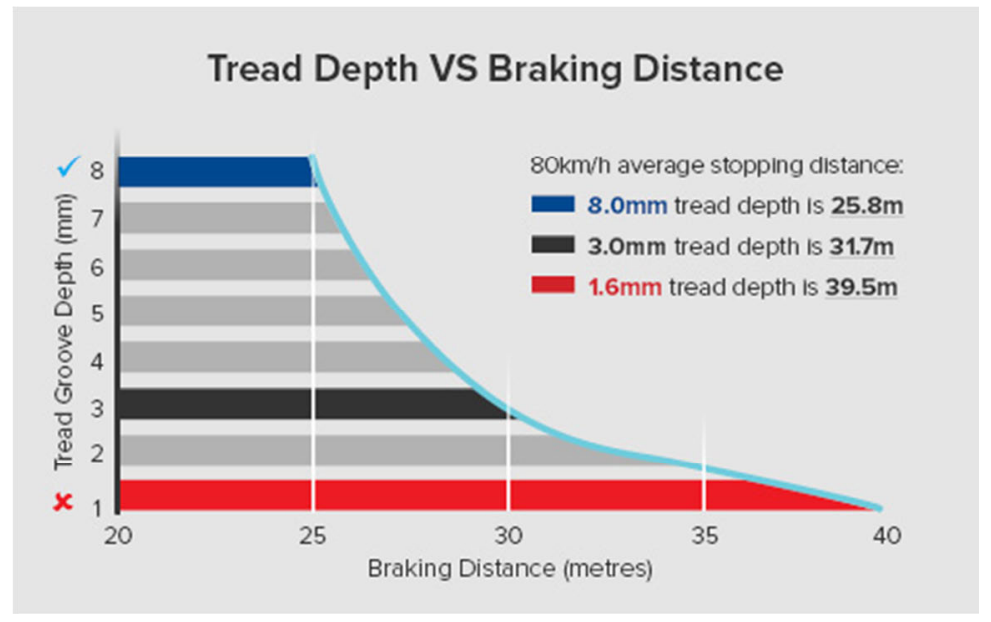

Fig. 1 Braking distance vs tread depth [10]

The tyre tread depth used to be determined by using manual contact measurements such as the so-called "penny test" or measurements performed by a tread depth gauge. The "penny test" gives a rough approximation of tread depth; the tyre is considered safe if the tread reaches a certain level on the penny, while the tread depth gauge gives an exact value of tread depth but it must be correctly positioned on a tread to determine its depth. These kinds of measurement are human factor-dependent, and inconvenient.

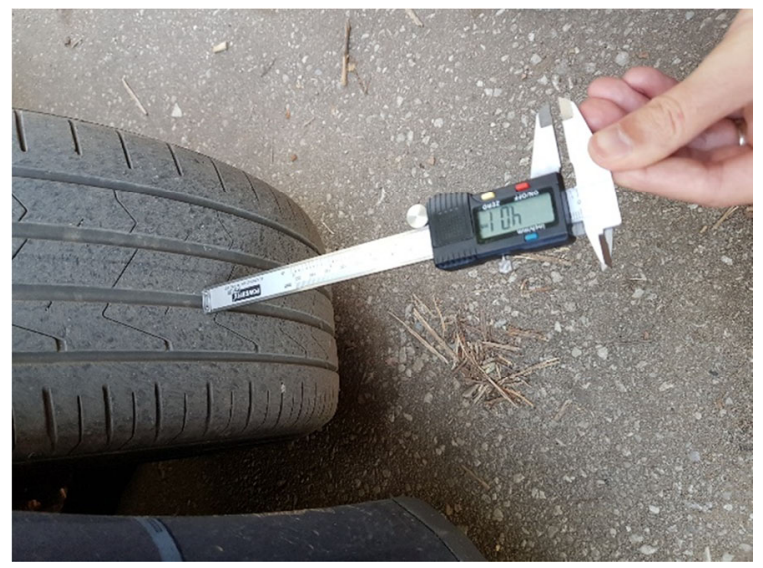

Fig. 2 The tyre tread depth measurement 
Several research projects have been carried out in the last few years with the aim to make the tyre tread inspection quicker, more accurate and more efficient as well as more independent of human labour.

Huang et al. [11] developed a machine vison-based prototype system to measure the tread depth by using a stereo camera. In the proposed system, based on the epipolar plane, the distance between the tyre treads and the camera lens was calculated; consequently, the image of the tyre tread depth was obtained. The obtained data are then transferred to the Android smart phone. The main disadvantage of this system is that the error in the determined tyre tread depth, in some cases, reached $1 \mathrm{~mm}$.

Jovančević et al. [12] proposed a new approach to inspect airplane tyres. The condition of tyres was inspected by investigating the tyre treads. Inspection results were obtained using the Ridge-based intensity profiling.

Wang et al. [9] developed a prototype system for tyre tread depth measurement based on machine vision. They used two cameras to capture an image of the profile of the tyre section at the intersection of the laser plane and the tyre. Then, the image was processed and calibrated to get the tyre section profile curve. The grooves on the tyre profile curve were then identified and the tyre tread depth was measured.

In addition to the research presented above, some researchers have been involved in research on how to detect tyre tread defects. For example, Chen et al. [13] inspected tyre tread defects by using image processing and pattern recognition methods, while Xiang et al. [14] used the dictionary-based representation to detect tyre defects.

In this paper, an automatic system for tyre tread inspection based on image processing and support-vector machine (SVM) classification is proposed. The idea is to develop a cheap and easy-to-implement system for automatic tyre inspection. The research on this subject is still in its early stage, and in this paper, only partial solutions are presented.

Experiments were performed with the aim to extract features from a segmented tyre and to determine which class the tyre belongs to. The idea is to classify a tyre according to the features of its tread pattern. Mostly used brands of summer season tyres in Serbia were taken into consideration. Precisely, our data set contains eight different types of tread patterns. If the tyre is worn, the surface of the tyre will be smoother, or the tyre treads may be gone. For that purpose, the Linear Support vector machine classifier was trained and tested. The investigated tyres were classified as a 'good tyre' class, which includes tyres with tread depths greater than $3 \mathrm{~mm}$, and a 'to replace' class, which includes tyres with tread depth of less than $3 \mathrm{~mm}$. Eighty percent of randomly chosen data from the whole data set was used to train the classifier, while $20 \%$ of data set was used for testing. Linear SVM has proven to be effective in the cases where the number of features is greater than the number of data points. The data set used in our experiments contains 103 images - 65 tyres in good condition and 38 worn out tyres. Both sets contain approximately the same number of different tyre tread types.

\section{Proposed methodology for the tyre tread depth classification}

A methodology for tyre tread depth classification proposed in this paper is shown in Fig. 3.

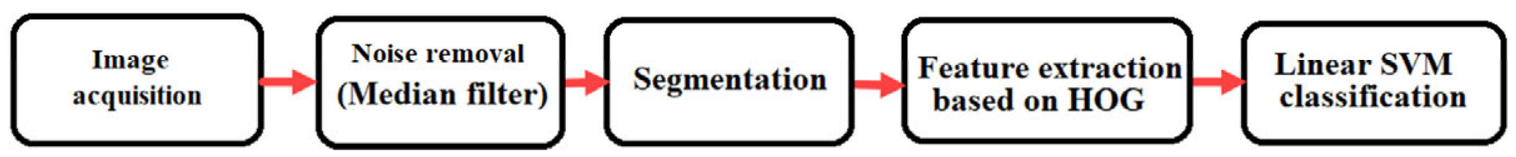

Fig. 3 The workflow of the system proposed for the tyre tread depth classification

For experimental purpose, images were captured by a mobile phone camera. 


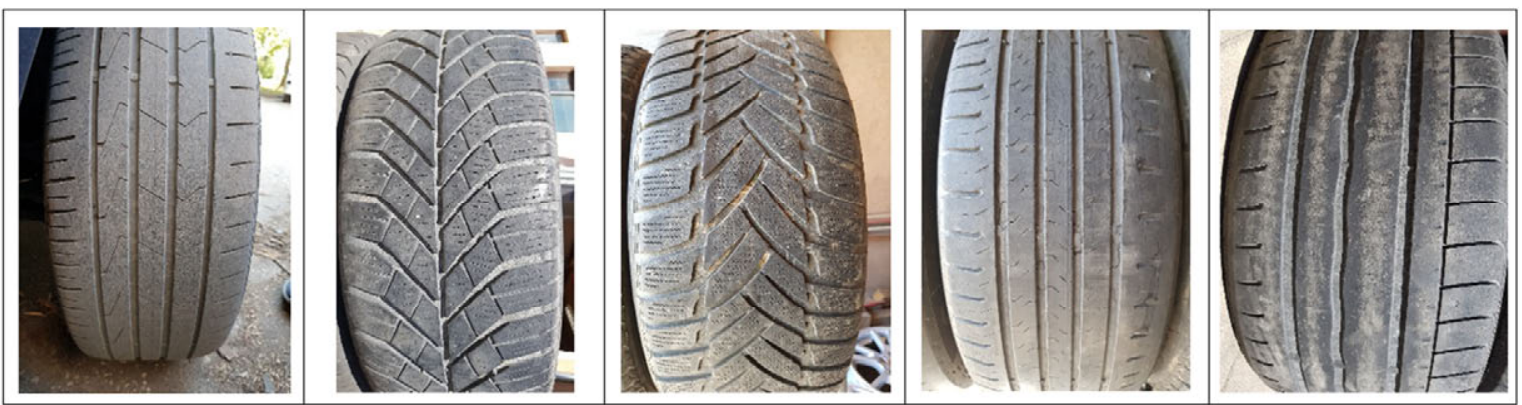

Fig. 4 Images used for experimental purposes (captured by a mobile phone camera)

\subsection{Median filter}

To reduce noise from captured images, a median filter was applied. The median filter belongs to the class of non-linear filters. It was introduced for the first time in 1997 [15] and has been widely used in image processing ever since. Furthermore, many image processing techniques, such as morphological processing, are just a variation of the basic median algorithm [16]. The main advantage of the median filter is its ability to preserve edges and small, sharp details while reducing image noise.

\subsection{Segmentation}

The first step in image analysis is image segmentation. The image segmentation separates objects of interest or regions of interest (RIO) from the other objects in the image so that the image can be read correctly and its content can be identified. In this experiment, the region of interest was user-determined by drawing a polygonal region. Then it was separated from the rest of the image.

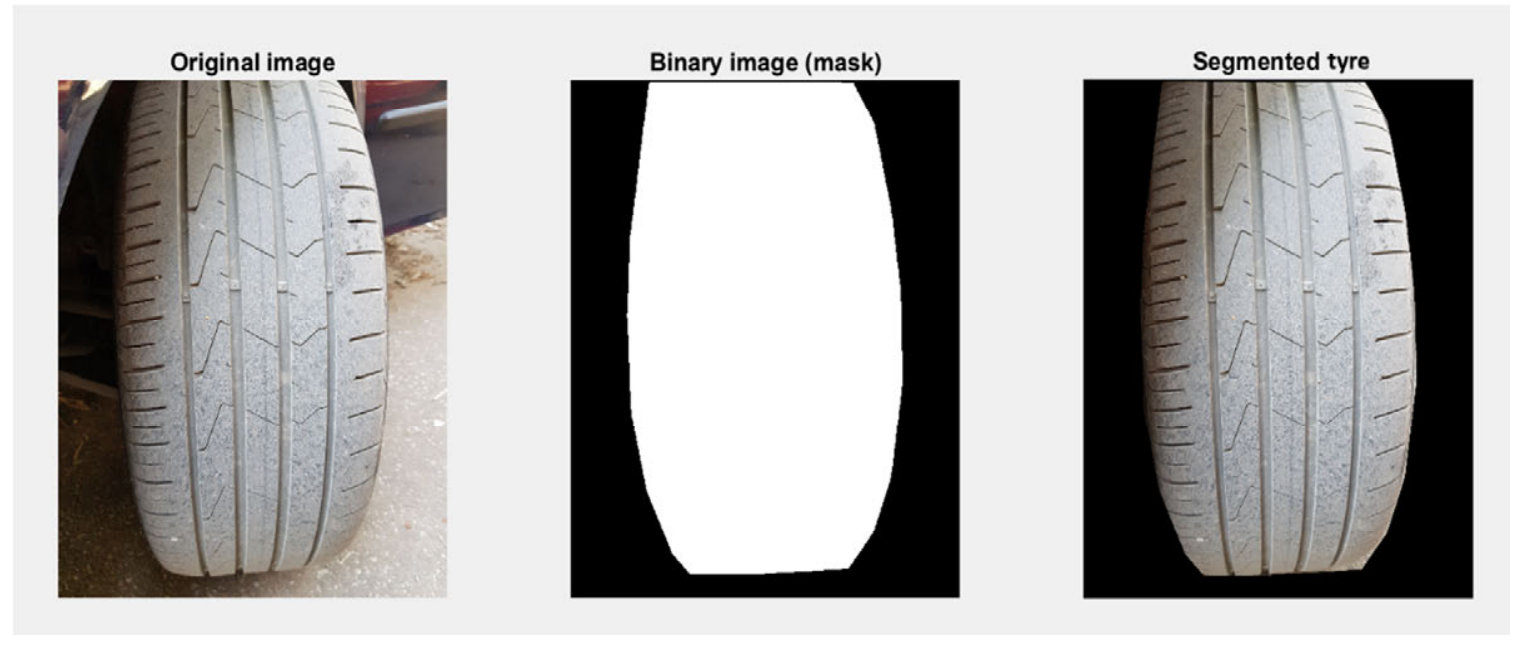

Fig. 5 User-determined RIO and a segmented object

After segmenting and separating the tyre from the rest of the image, the characteristics describing the segmented region are calculated by using the histogram of oriented gradients.

\subsection{Feature extraction based on the histogram of oriented gradients (HOG)}

Edges and their orientations play a significant role in describing and recognizing objects in the image [17]. The main idea of using HOG, introduced by Dalal and Triggs [18], is that the appearance of an object can be described by the local distribution of its edges and their orientations. Additionally, these orientations are calculated in 'localized' portions by dividing the image into smaller regions and calculating the gradients and orientation of each region. 


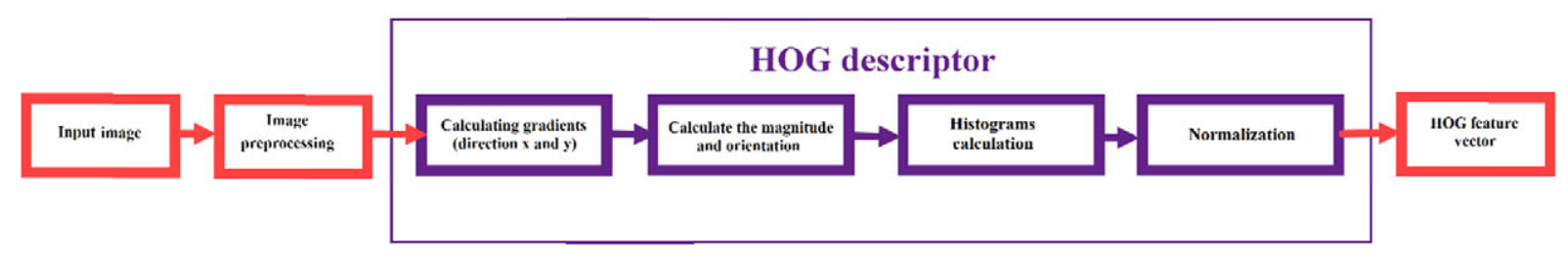

Fig. 6 A diagram of the feature extraction system using HOG

Pre-processing is an inevitable step in image processing or more generally in any machine learning project.

To compute the HOG descriptor, it is necessary to pre-process the image and resize the width and height of the image to a 1:2 ratio, respectively. This is because the image is usually divided into $8 \times 8,16 \times 16$ or $32 \times 32$ pixel cells to extract the features. In this research, the image size is set to be $128 \times 256$ pixels. The next step is to compute the gradient vector of every pixel, as well as its magnitude and orientation. After that, the image is divided into cells, and for each cell, a histogram of oriented gradients is computed by binning and cumulatively adding the magnitude values of that cell into a certain number of buckets.

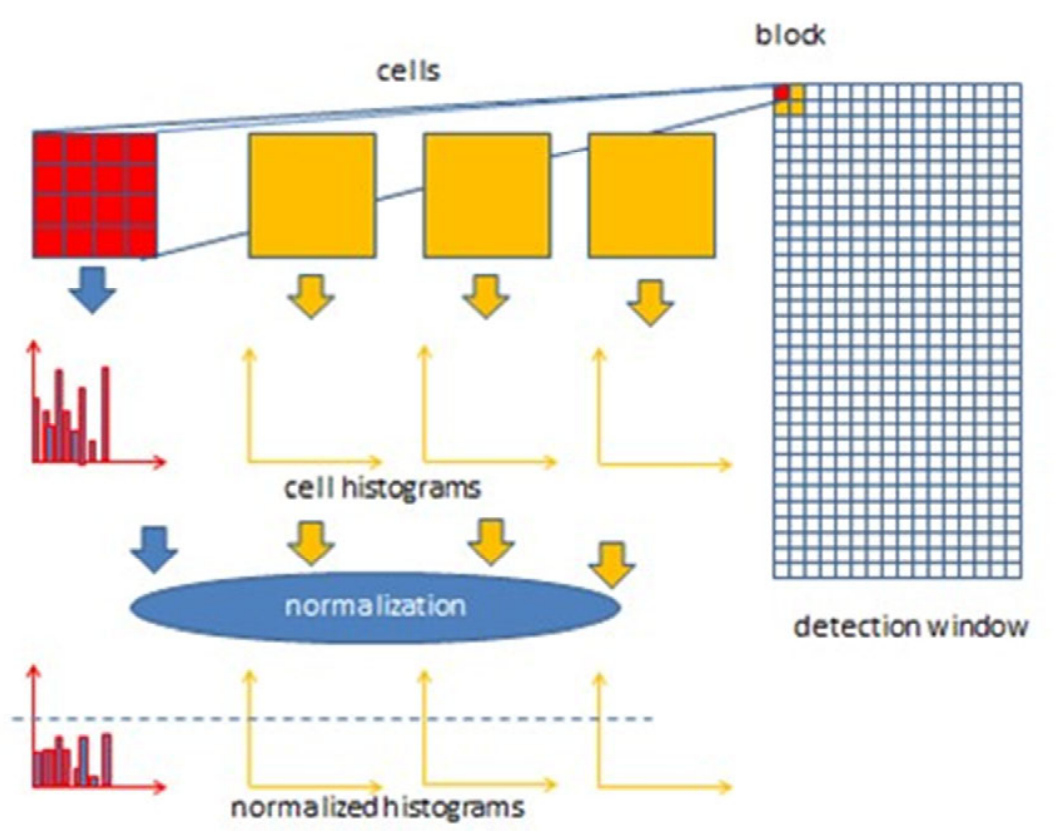

Fig.7 Workflow of the histogram of oriented gradients [19]

Although the HOG features are created, the gradients of the image are sensitive to the overall lighting, which leads to some parts of the image to be brighter compared to the rest. To reduce this lighting variation, instead of normalizing each histogram individually, the cells are grouped into blocks and histograms are normalized based on all histograms in the block. The resulting vector is the concatenation of the normalized blocks.

Thus, when applying HOG, we can set the following parameters:

- the number of buckets we want to create. A practical choice based on empirical experiments is 9 buckets, and in this paper the same number of buckets is used.

- the number of pixels per cell. This number defines the size of the cell for which the histograms are created.

- the number of cells per block. This number is the size of the block over which we normalize the histogram. 
Different sizes of the cell and blocks lead to different numbers of extracted features. For example, for a single $128 \times 256$ image, if we chose $8 \times 8$ pixels per cell and $2 \times 2$ cells per block, we have $465(15 \times 31)$ blocks of $16 \times 16$ cells. Each of these 465 blocks has a vector of 36 features $(9 \times 4)$. Therefore, the total number of features for the image would be $465 \times 36$, which gives 16740 features.

In order to evaluate the effects of various numbers of features on the classifier efficacy, and thus to choose the optimal set of HOG parameters, we carried out experiments in which the linear SVM classifier was trained and tested with different numbers of features.

Figure 8 shows the HOG characteristics of a good and a worn out tyre of the same pattern. The HOG parameters are $8 \times 8$ pixels per cell and $2 \times 2$ cells per block.

a)
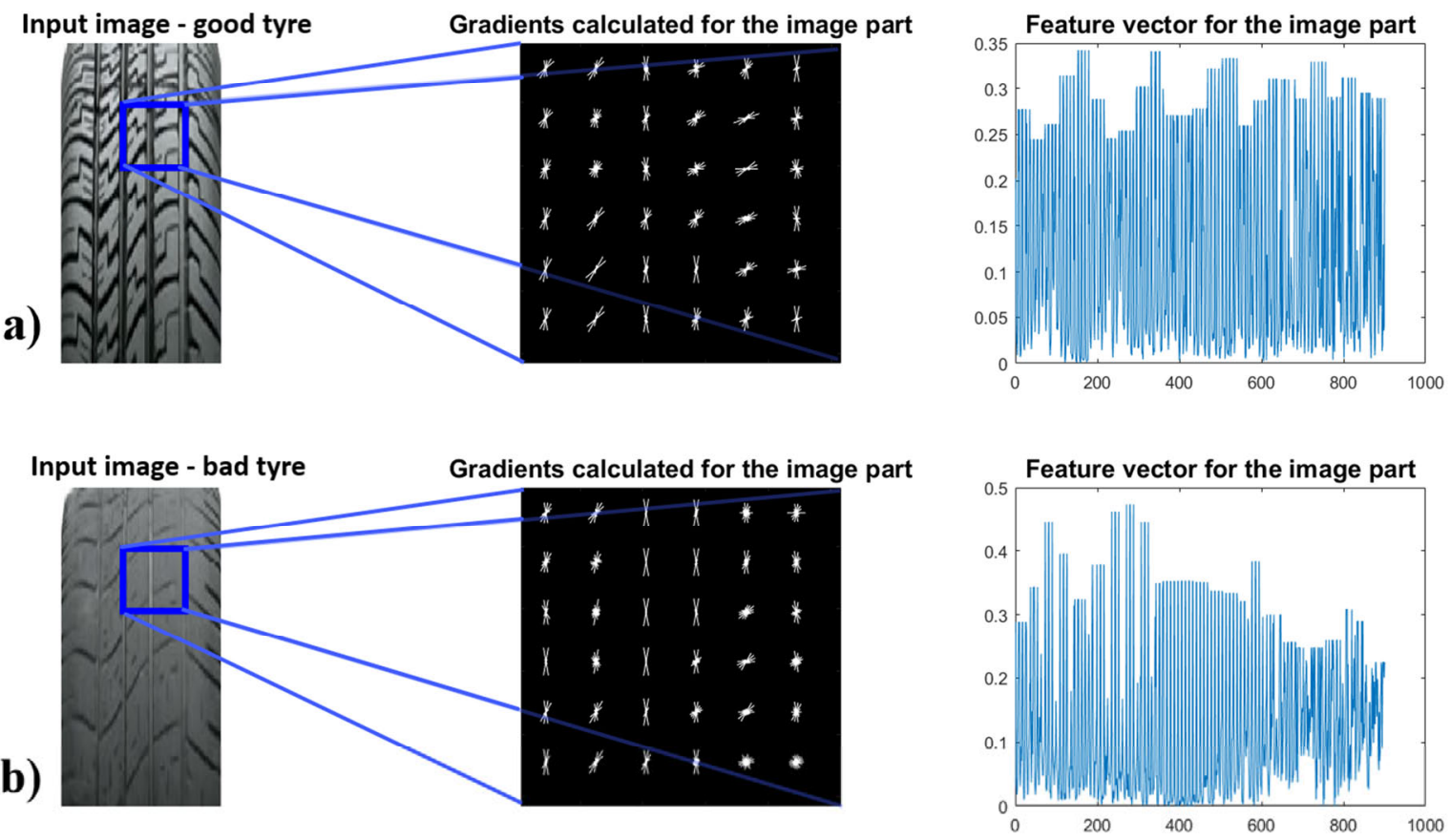

Fig. 8 Comparison of feature extraction results obtained using HOG; a) Input image is a good tyre;

b) Input image is a bad tyre

\subsection{Linear SVM classification}

The support-vector machine is a machine learning methodology which has proven to be excellent in pattern recognition, especially in supervised classification of high-dimensional data sets [20]. For a given a set of $m$ training data points, each with $n$ features and an associated class label, the SVM attempts to separate these points using an (n-1)-dimensional hyperplane [21]. There are many possible hyperplanes that could be used to separate the data into classes. The objective is to find the hyperplane that has the maximum margin-maximum distance between the dividing hyperplane and any given data point. As the margin distance increases, the future data points are classified with a higher accuracy.

A linear support-vector machine aims to construct a hyperplane:

$$
\boldsymbol{w}^{T} \boldsymbol{x}+b=\sum_{i=1}^{n} w_{i} x_{i}+b
$$

where $\boldsymbol{w}$ represents the weight vector, $\boldsymbol{x}$ is the feature vector, and $b$ is the bias. 
The optimal weight vector $\boldsymbol{w}$ and the bias $b$ are obtained by solving the following optimization problem:

$$
\min _{\boldsymbol{w}, b, \xi} \frac{1}{2}\|\boldsymbol{w}\|^{2}+C \sum_{i=1}^{m} \xi_{i}
$$

The slack variables $\zeta_{\mathrm{i}}$ are added to constrain the optimization problem. The constraints now become $y_{i}\left(w \cdot x_{i}+b\right) \geq 1-\zeta_{i}, i=1 \ldots m$

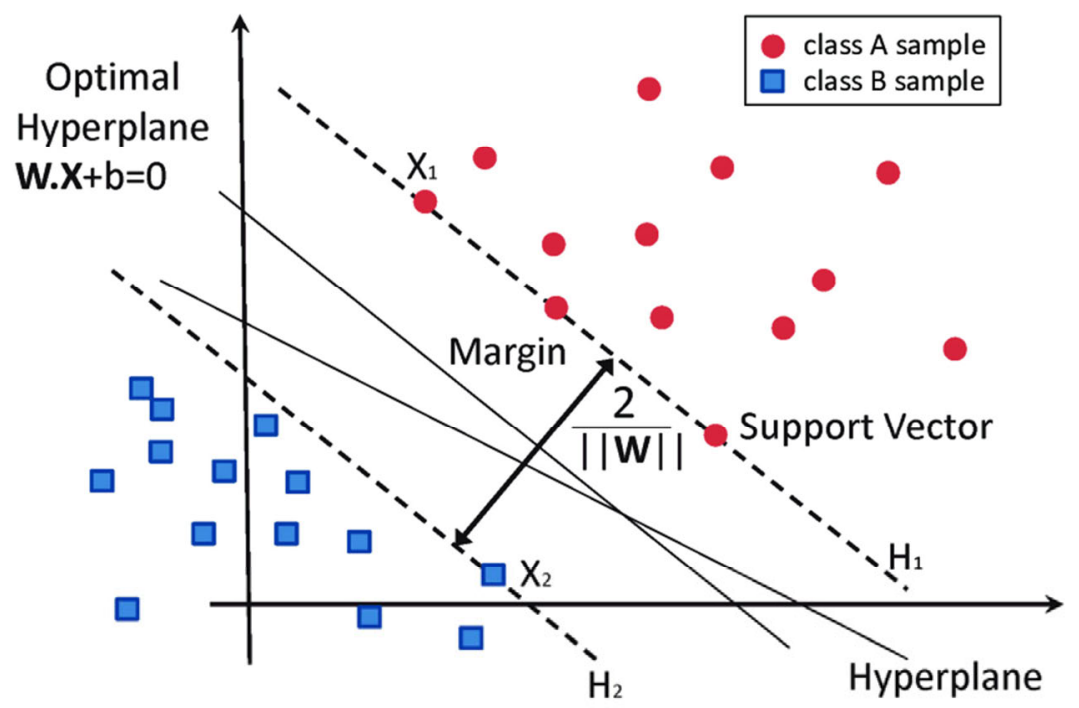

Fig. 9 Binary classification of data by support-vector machine [21]

The regularization parameter $\mathrm{C}$ is a user-adjustable parameter used to designate the importance of $\zeta$, i.e. to determine how much we want to avoid misclassification of each training example.

By introducing the Lagrange multipliers $\alpha_{i}$ to Eq. (2), the optimization problem is transformed into a dual problem:

$$
\max _{\alpha} \sum_{i=1}^{m} \alpha_{i}-\frac{1}{2} \sum_{i, j=1}^{m} \alpha_{i} \alpha_{j} y_{i} y_{j} x_{i} \cdot x_{j}
$$

subject to $0 \leq \alpha_{i} \leq C, \underset{i=1}{m} \alpha_{i} y_{i}=0$

In solving this problem, we only care about the result of the dot product $x_{i} \cdot x_{j}$. Hence, a function for calculating the dot product has been introduced into the equation. This function is called a kernel function. The result we get is the same as the result we get when the data is transformed into a higher dimension.

The linear kernel is defined as $K\left(x_{i}, x_{j}\right)=x_{i}^{T} x_{j}$.

Finally, the decision function for classifying a new data sample $\hat{x}$ is:

$$
\hat{\mathrm{y}}=\operatorname{sgn}\left(\sum_{i=1}^{m} y_{i} \alpha_{i} K\left(\boldsymbol{x}_{i}, \hat{\boldsymbol{x}}\right)+b\right)
$$

\section{Results}

To analyse which set of HOG features gives the best classification results, the linear SVM classifier was trained and tested using different values for the number of pixels per cell $(8 \times 8,16 \times 16$, and $32 \times 32)$ and the number of cells per block $(2 \times 2$ and $4 \times 4)$. Efficiency of the trained models is evaluated using two metrics: accuracy and $\mathrm{F}_{1}$-score. 
Table 1 Accuracy and $\mathrm{F}_{1}$-score values for different sets of HOG parameters

\begin{tabular}{|c|c|c|c|c|}
\hline $\begin{array}{c}\text { CELLS PER } \\
\text { BLOCK }\end{array}$ & $\begin{array}{c}\text { PIXELS } \\
\text { PER CELL }\end{array}$ & $\begin{array}{c}\text { NUMBER OF } \\
\text { FEATURES }\end{array}$ & $\begin{array}{c}\text { ACCURACY } \\
\text { (\%) }\end{array}$ & F $_{1-S C O R E}$ \\
\hline \multirow{4}{*}{$2 \times 2$} & $8 \times 8$ & 16740 & 99 & 0.9922 \\
\cline { 2 - 5 } & $16 \times 16$ & 3780 & 99 & 0.9922 \\
\cline { 2 - 5 } & $32 \times 32$ & 756 & 92.2 & 0.9375 \\
\hline \multirow{3}{*}{$4 \times 4$} & $8 \times 8$ & 15120 & 99 & 0.9922 \\
\cline { 2 - 5 } & $16 \times 16$ & 3024 & 96.1 & 0.9697 \\
\cline { 2 - 5 } & $32 \times 32$ & 432 & 87.4 & 0.8943 \\
\hline
\end{tabular}

Based on the data shown in Table 1, we decided to choose the HOG with 16x16 pixels per cell and $2 \times 2$ cells per block because it has the same accuracy and the $\mathrm{F}_{1}$-score as HOGs with $8 \times 8$ pixels, but it has 4 times smaller number of extracted features. Also, with a smaller number of features, the classification of new data will be faster, which is important for realtime applications. For example, the prediction time for data with 16740 features $(8 \times 8$ pixels per cell and $2 \times 2$ cells per block) is $3.2346 \mathrm{~s}$, while the prediction time for data with 3780 features (16x16 pixels per cell and $2 \times 2$ cells per block) is $0.2579 \mathrm{~s}$.

Figure 8 shows a confusion matrix for the linear SVM classifier trained with a data set with 3780 features.
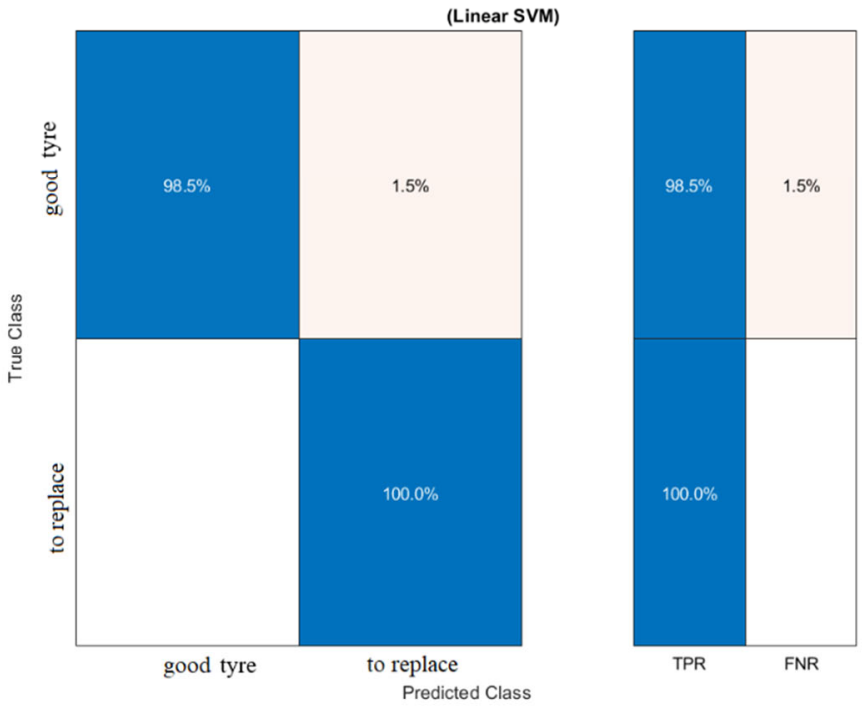

Fig. 10 Confusion matrix

In Fig. 10 we can see that the true positive rate for the trained SVM classifier is $98.5 \%$, while the true negative rate is $100 \%$. This means that the classifier did not fail to classify the tyre that should be replaced, but in $1.5 \%$ of cases, the tyre from the "good tyre" class was classified into the "to replace" class. It should be mentioned that in the training data, a few tyres from the "good tyre" class had the tread depth slightly greater than $3 \mathrm{~mm}$, so it can be concluded that the obtained results are encouraging.

Additionally, to test the classifier robustness against various impurities which can occur on tyres, some tests were done. The tyre that belongs to the "good tyre" class was treated with different impurities. The experiment was performed on the tyre stained with sand, waterlogged mud, and sodden mud (Fig. 11). 
a)

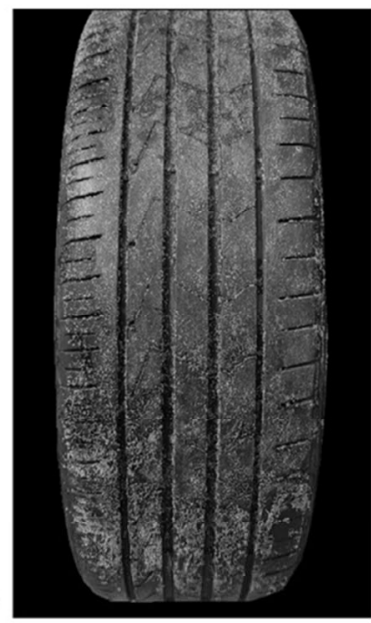

b)

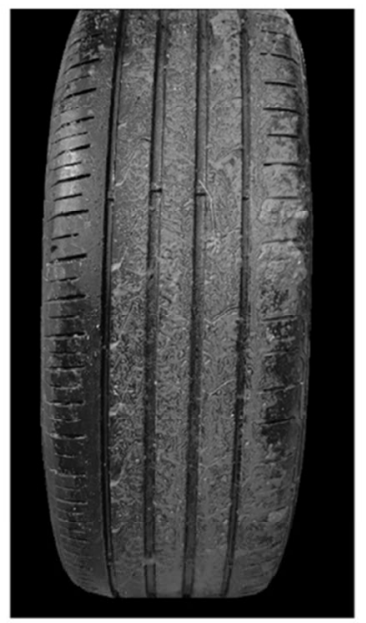

c)

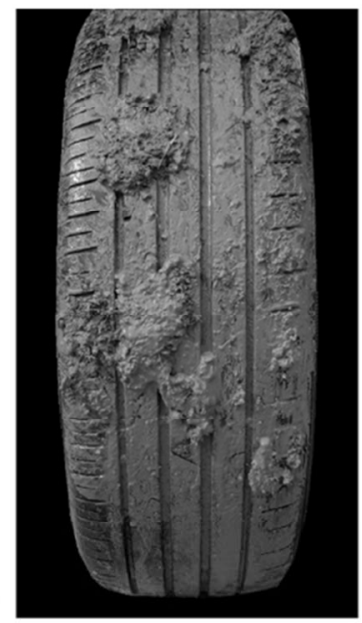

Fig. 11 The segmented tyre with different kinds of impurities: a) sand, b) waterlogged mud, c) sodden mud

The classifier was able to classify the tyre in the appropriate class, which is promising for feature research.

\section{Conclusion and future work}

In this paper, an approach for visual, non-contact, automatic tyre tread depth inspection based on existing image processing techniques is presented. The proposed method applies the histogram oriented gradient and a support-vector machine for the tyre tread depth inspection. The obtained classification results with an accuracy of $99 \%$ and an $\mathrm{F}_{1}$-score of 0.9922 are very promising, even with a relatively small number of samples.

The testing of classifier robustness has also given encouraging results. Further experiments will include an increased data set and more tests with different types of tyres and various impurities which may be found on them. Also, future research will include the detection of objects such as nails which damage tyres and make them unsafe.

In this paper, the region of interest (ROI) is determined manually, by drawing a polygonal region. The focus of our future wok will be primarily on automatic segmentation and determination of region of interest as a necessary part of an automatic system for tyre tread inspection.

\section{REFERENCES}

[1] Dugoff, H.; Fancher, P.S; Segel, L. Tire performance characteristics affecting vehicle response to steering and braking control inputs, Highway Safety Research Institute 1969, University of Michigan.

[2] Sivaramakrishnan, S; Singh, K.B; Lee, P. Influence of Tire Operating Conditions on ABS Performance, Tire Science and Technology 2015, 43(3): 216-241. https://doi.org/10.2346/tire.15.430302

[3] Bras, B.; Cobert, A. Life-cycle environmental impact of Michelin Tweel ${ }^{\circledR}$ tire for passenger vehicles, SAE international journal of passenger cars-mechanical systems 2011, 4(1):32-43. https://doi.org/10.4271/2011-01-0093

[4] Höpping, K.; Augsburg, K. Dynamic tire pressure control system-analysis of the effect to longitudinal vehicle dynamics and fuel consumption. In: Conference: Shaping the future by engineering: 58th IWK, Ilmenau Scientific Colloquium At: Ilmenau; 2014.

[5] Du, Z.; Wen, X.; Zhao, D.; Xu, Z.; Chen, L. Numerical analysis of partial abrasion of the straddle-type monorail vehicle running tyre, Transactions of FAMENA 2017, 41(1): 99-112. https://doi.org/10.21278/TOF.41109

[6] Hobeika, T.; Sebben, S.; Landstrom, C. Investigation of the influence of tyre geometry on the aerodynamics of passenger cars, SAE International Journal of Passenger Cars-Mechanical Systems 2013, 6(1): 316-325. https://doi.org/10.4271/2013-01-0955

[7] Siegel, J.; Sun, Y.; Sarma, S. Automotive Diagnostics as a Service: An Artificially Intelligent Mobile Application for Tire Condition Assessment, In: Artificial Intelligence and Mobile Services - AIMS 2018 2018, p.172-184. https://doi.org/10.1007/978-3-319-94361-9_13. 
[8] Cowley, J.A.; Kim, S.; Wogalter, M.S. People do not identify tire aging as a safety hazard, In: Proceedings of the Human Factors and Ergonomics Society Annual Meeting 2006, 50(8): 860-864. Sage CA: Los Angeles, https://doi.org/10.1177/154193120605000805

[9] Wang, X.B.; Li, A.J.; Ci, Q.P.; Shi, M.; Jing, T.L.; Zhao, W.Z. The study on tire tread depth measurement method based on machine vision, Advances in Mechanical Engineering 2019,11(4), https://doi.org/10.1177/1687814019837828

[10] Data Source: Courtesy of the British Rubber Manufacturers' Association (UK). Test carried out by independent experts, the Motor Industry Research Association (UK). https://www.coopertires.com.au/cooper-life/blog/2018/march/worn-out-tyres

[11] Huang, S.Y.; Chen, Y.C.; Wang, J.K. Measurement of tire tread depth with image triangulation, In: International Symposium on Computer, Consumer and Control (IS3C) 2016, 303-306. Publisher: IEEE https://doi.org/10.1109/IS3C.2016.86

[12] Jovančević, I.; Arafat, A.; Orteu, J.J.; Sentenac, T. Airplane tire inspection by image processing techniques, In: 5th Mediterranean Conference on Embedded Computing (MECO) 2016, p. 176-179. Publisher: IEEE, https://doi.org/10.1109/MECO.2016.7525733

[13] Chen, P.; Shubinsky, G.D.; Jan, K.H.; Chen, C.A.; Sidla, O.; Poelzleitner W. Inspection of tire tread defects using image processing and pattern recognition techniques, In: Proc. SPIE 2063, Vision, Sensors, and Control for Automated Manufacturing Systems 1993, https://doi.org/10.1117/12.164968

[14] Xiang, Y.; Zhang, C.; Guo, Q. A Dictionary-based Method for Tire Defect Detection, In: IEEE International Conference on Information and Automation (ICIA), Hailar, China, 2014, p. 519 - 523. https://doi.org/10.1109/ICInfA.2014.6932710

[15] Tukey, J.W. Exploratory data analysis, Publisher: Reading, Mass.: Addison-Wesley Pub. Co., 1977.

[16] Weiss, B. Fast median and bilateral filtering, In: ACM Transactions on Graphics 2006, p. 519-526. https://doi.org/10.1145/1141911.1141918

[17] Ballesteros, G.; Salgado, L. Histograms of oriented gradients for fast on-board vehicle verification, In: 2014 IEEE International Conference on Image Processing (ICIP), 2014, p.1638-1642. https://doi.org/10.1109/ICIP.2014.7025328

[18] Dalal, N.; Triggs, B. Histograms of oriented gradients for human detection, In: 2005 IEEE computer society conference on computer vision and pattern recognition (CVPR'05), 2005, p. 886-893. https://doi.org/10.1109/CVPR.2005.177

[19] Prashar, K.; Talwar, R.; Kant, C. Robust automatic cotton crop disease recognition (ACDR) method using the hybrid feature descriptor with SVM, In: 4th 2016 International Conference on Computing on sustainable Global Development, 01.03-03.03.2017. Paschim Vihar, New Delhi.

[20] Tzotsos, A.; Argialas D. Support vector machine classification for object-based image analysis, In: Object-Based Image Analysis 2018, Springer, Berlin, Heidelberg; p. 663-677, https://doi.org/10.1007/978-3-540-77058-9_36

[21] Ma, Y.; Chen, W.; Ma, X.; Xu, J.; Huang, X.; Maciejewski, R.; Tung, A.K. Easy SVM: A visual analysis approach for open-box support vector machines, Computational Visual Media 2017, 3(2):161-175. https://doi.org/10.1007/s41095-017-0077-5

[22] García-Gonzalo, E.; Fernández-Muñiz, Z.; García Nieto, P.J; Bernardo Sánchez, A.; Menéndez Fernández M. Hard-rock stability analysis for span design in entry-type excavations with learning classifiers, Materials 2016, 9(7): 531. https://doi.org/ 10.3390/ma9070531

Submitted: $\quad 14.11 .2020$

Accepted: $\quad$ 05.7.2021
Emina Petrović*

University of Niš, Faculty of Mechanical Engineering, Aleksandra Medvedeva 14, 18000 Niš, Serbia

Danijela Ristić Durrant Institute of Automation, University of Bremen, NW1/ FB1

Otto-Hahn-Allee 1 28359 Bremen, Germany

Miloš Simonović

Žarko Ćojbašić

Vlastimir Nikolić

University of Niš, Faculty of Mechanical Engineering, Aleksandra Medvedeva 14, 18000 Niš, Serbia ${ }^{*}$ Corresponding author: emina.petrovic@masfak.ni.ac.rs 